\title{
A citizen science project reveals contrasting latitudinal gradients of wing deformity and parasite infection of monarch butterflies in New Zealand
}

\author{
P H I L I P J L E S T E R (D) and M A R I A N A B U L G A R E L L A (1) School of Biological \\ Sciences, Victoria University of Wellington, Wellington, New Zealand
}

\begin{abstract}
Host-parasite interactions represent complex relationships among species, often with considerable spatial variation. We examined latitudinal variation in the prevalence of monarch butterfly (Danaus plexippus) infection by the protozoan parasite Ophryocystis elektroscirrha via a citizen science project in New Zealand. Parasitism by $O$. elektroscirrha can result in incomplete development causing mortality, or adult butterflies with deformed wings and reduced fitness.

2. We established the existence of counter-latitudinal clines of wing deformities and parasite prevalence in monarchs, which in New Zealand are non-migratory. The prevalence of wing deformities increased with increasing latitude, whereas the prevalence of parasite infections decreased with increasing latitude. No parasitism was observed in our highest latitudinal location, while all butterfly samples were infected in the warmest and lowest latitudinal site. The prevalence of parasitism or wing deformity did not vary with the butterfly sex.

3. Cold temperatures appear to independently affect the parasite and host butterfly development, with lower temperatures limiting parasite development and inducing wing deformities in butterflies. Other protozoan parasites display similar latitudinal clines that are thought to be temperature related.

4. Environmental factors appear to independently influence insect populations through different mechanistic pathways. The high prevalence of parasite infections may affect butterfly fitness in warmer regions, but the cooler conditions at higher latitudes manifest as a major fitness cost, deformed wings. Monarch butterflies in New Zealand and around the world may perform better where it is not too hot (and optimal for these parasites), but not cold (where the pupae experience developmental issues).
\end{abstract}

Key words. Danaus plexippus, deformity, Ophryocystis elektroscirrha, parasitism, temperature.

\section{Introduction}

Host-parasite interactions represent common albeit complex relationships among species (Musgrave et al., 2019). Parasite richness, prevalence, and the outcome of the host-parasite interactions vary significantly between geographical regions (Dunn et al., 2010). For many plants and animals, species richness and abundance peak in the tropics and decrease towards the poles. Spatial variation in parasitism is well documented in a variety of taxa including trees (Albrectsen et al., 2010), insects

Correspondence: Mariana Bulgarella, School of Biological Sciences, Victoria University of Wellington, PO Box 600, Wellington,

New Zealand. E-mail: mariana.bulgarella@vuw.ac.nz
(Webberley et al., 2006; Corby-Harris \& Promislow, 2008), crustacean (Wolinska et al., 2011), fish (Rohde, 1993, 2002; Timi, 2003), birds (Calvete et al., 2003; Loiseau et al., 2010), and mammals (Cumming, 2000; Nunn et al., 2005). For marine fish, for example, the diversity of monogenean parasites increases with decreasing latitude or increasing water temperature (Rohde, 1993; Rohde \& Heap, 1998). Explanations for these patterns include the energy hypothesis and the evolutionary time hypothesis (reviewed by Rohde, 1999; Poulin, 2007). These processes may act in synergy.

Monarch butterflies (Danaus plexippus) are an iconic and popular species, which are parasitised by the neogregarine protozoan Ophryocystis elektroscirrha in their native and introduced 
ranges (McLaughlin \& Myers, 1970; Leong et al., 1997). This parasite is a specialist on Danaus spp. (Gao et al., 2020). It can harm butterflies by negatively affecting several life-history parameters including pre-adult survival, adult body mass, mating ability, fecundity, flight ability, and adult lifespan (de Roode et al., 2019). Heavy infections appear to impair normal development leading to crippling wing deformities (Bradley \& Altizer, 2005; de Roode et al., 2008a). Parasitism by O. elektroscirrha has been hypothesised to be a contributing factor to monarch butterfly declines (Davis \& Rendon-Salinas, 2010; Thogmartin et al., 2017). It is also thought to have contributed to the evolution of monarch migratory behaviour. The 'migratory escape' hypothesis predicts that migrating butterflies benefit from escaping pathogen accumulation in contaminated environments (Bartel et al., 2011; Flockhart et al., 2018; de Roode et al., 2019). The 'migratory culling' hypothesis predicts the selective removal of infected butterflies occurs during their migration (Bradley \& Altizer, 2005; Bartel et al., 2011; de Roode et al., 2019). Debate continues as to how parasitism by O. elektroscirrha has contributed to the evolution of migratory behaviour in these butterflies.

Monarch butterflies have expanded their distribution to at least 74 countries around the world (Nail et al., 2019). They are the only resident Danaus species and host to O. elektroscirrha in New Zealand, although D. chrysippus and D. hamatus are infrequently blown to the country from Australia (Clarke \& Zalucki, 2004; Zalucki \& Clarke, 2004; Gibbs, 2013). In New Zealand, monarch butterflies occupy a latitudinal range of -34.5 to $-46.0^{\circ} \mathrm{S}$ (Gibbs, 1980), which encompasses a wide array of temperatures and growing conditions.

Temperature seems the most critical factor affecting Lepidoptera dynamics (Li et al., 2017; Thogmartin et al., 2017). Protozoan parasites in other species have previously been observed to vary with latitude owing to their sensitivity to factors such as temperature, $\mathrm{pH}$, rainfall, and host diversity and abundance (Zilberstein \& Shapira, 1994; Harvell et al., 2002). In addition, temperature-dependent effects on parasite abundance and virulence have been widely observed (Dawson et al., 2005; Scharsack et al., 2016; Musgrave et al., 2019).

The New Zealand monarch populations offer a simplification of the tritrophic system present in their home range in North America. Serial founder effects have resulted in monarch populations displaying low genetic diversity (Pierce et al., 2014). The limited diversity of these butterflies likely also limits the number of parasite genotypes, which have been shown to have varying levels of virulence in North America (de Roode et al., 2007). There are at least 33 different milkweed plants in North America that can alter the parasite-host relationship (de Roode et al., 2008b; Nail et al., 2019), but only four milkweed species occur in New Zealand. Two species of Gomphocarpus (G. fructicosus and G. physocarpus) were introduced from South Africa and two Asclepias species (A. curassavica and A. incarnata) were introduced from North America. With the exception of A. incarnata, which loses its leaves over winter, the other three milkweed species are perennial in New Zealand, with A. incarnata being very uncommon (Gibbs, 2013). These shrubs are usually grown in gardens but they can also propagate naturally (Gibbs, 2013). Additionally, monarch butterfly populations in
New Zealand are non-migratory with one study finding $<3 \%$ of individuals fly more than $20 \mathrm{~km}$ (Wise, 1980).

One previous study found that $O$. elektroscirrha parasitism occurs worldwide in nearly every monarch population examined (Altizer \& de Roode, 2015). That survey included data from New Zealand, and showed that $O$. elektroscirrha was present, at approximately $50 \%$ infection prevalence, albeit a sample size of just six individuals. In our study, we sampled monarch butterflies via a citizen science project. Previous studies have similarly used citizen scientists to sample and help understand this host-parasite relationship (Bartel et al., 2011; Satterfield et al., 2015, 2016) and many other aspects of monarch biology (Ries \& Oberhauser, 2015).

The objectives of our study were to determine the prevalence of O. elektroscirrha infections in monarch butterflies across New Zealand, and test whether wing deformities are correlated with parasitism rates. Our initial prediction was that higher parasite loads will have a higher fitness cost that can be phenotypically observed as crumpled or deformed wings. We also asked if the monarch-O. elektroscirrha interaction varied significantly by latitude, a proxy for air temperature.

\section{Materials and methods}

We sampled butterflies during the austral autumn of 2020. Autumn is when high numbers of $O$. elektroscirrha spores occur (Bartel et al., 2011) and coincided with the observation of a high number of wing deformities and monarch pupae having difficulties emerging from their chrysalis (Fig. 1a,b). We developed a website and press release asking for samples from New Zealand citizens (Young, 2020). Citizens were asked to sample any adult monarch butterflies present in their gardens. Sampling O. elektroscirrha can be harmlessly and easily achieved by placing clear sticky tape (e.g. Scotch Tape) on the adult butterfly abdomen, to which a combination of butterfly scales and distinctive parasite spores stick (Altizer et al., 2000; Davis et al., 2004) (Fig. 1c,d). The spores can only be seen under $>50 \mathrm{x}$ magnification. Citizens were asked to mail the tape with information on the wing condition (normal or deformed), butterfly sex, sampling location, and whether the samples were taken from wild or reared butterflies to the university. Many submitters also emailed us photographs of the butterflies showing the wing deformities. Not all submitted samples contained information on butterfly sex, wing deformity, or rearing information, so our sample size is different in different analyses. Our request coincided with the lockdown period for New Zealand during the COVID-19 virus pandemic and we had a total of 97 individuals/families, aged between 4 and 86 submit samples. Sampling took place between 15 April 2020 and 27 July 2020.

Following previous research (Altizer et al., 2000; de Roode et al., 2009; Flockhart et al., 2018), we quantified spore counts into four categories based on the transmission biology of the parasite: $0=$ no spores present, $1=1-99$ spores, i.e., mild infection likely due to contamination as adults via mating or other activity (see de Roode et al., 2007, 2009; Majewska et al., 2019), $2=100-499$ spores, medium infection and $3=$ $>500$ spores, heavy to very heavy infection (Table S1). Thus, 


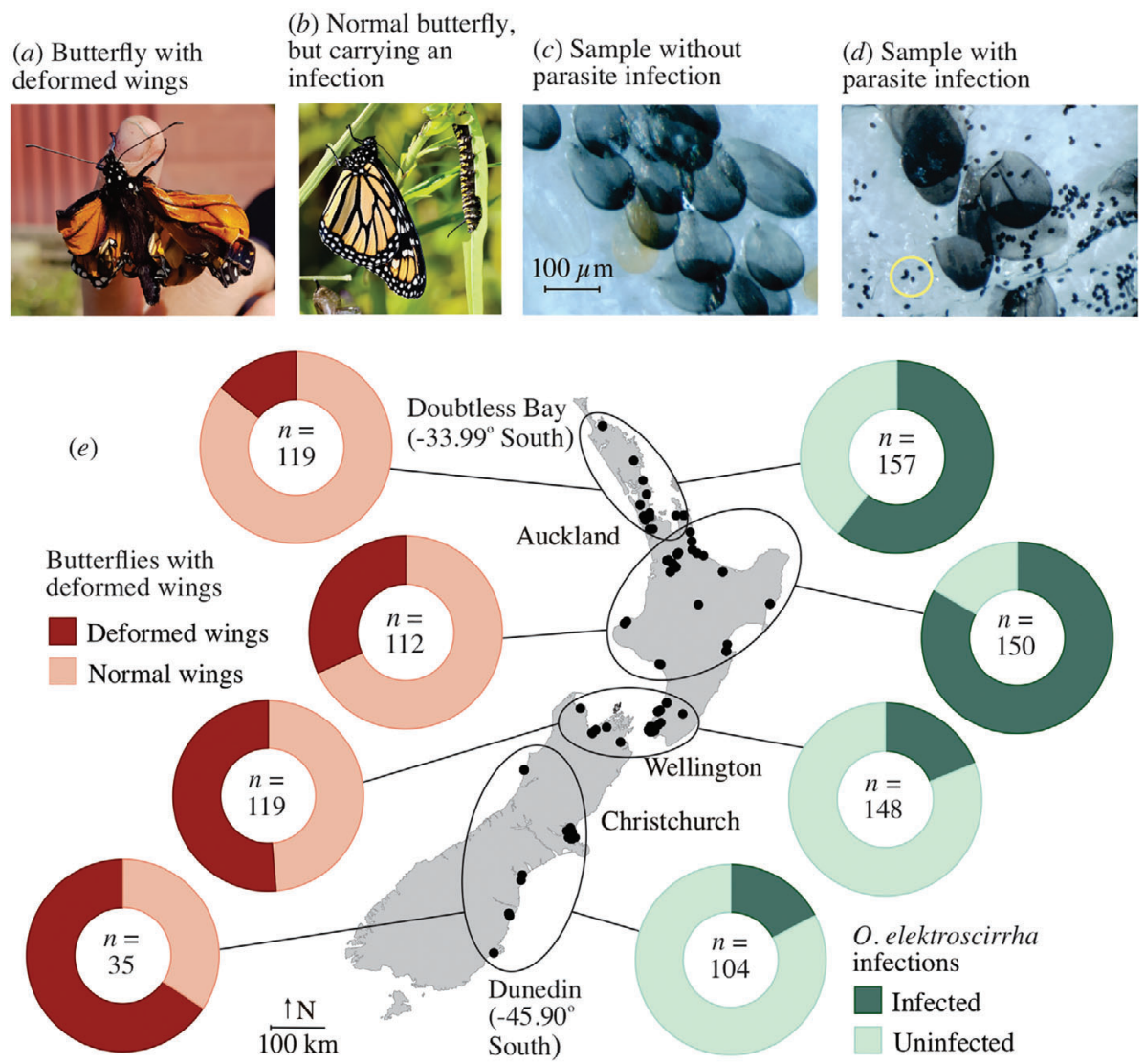

Fig. 1. (a) A butterfly with crumpled wings raised by 6-year-old Nicolas of Rotorua (photograph by Nicky King); (b) this adult butterfly has normal wings, but observed to be heavily infected by the protozoan parasite Ophryocystis elektroscirrha; (c) an example of samples showing butterfly scales but no spores, and (d) butterfly scales along with $O$. elektroscirrha spores (circled in yellow); (e) a map of New Zealand with pie charts showing deformed wing or infection prevalence. $n=$ sample size; some people did not indicate the wing status of their butterfly samples (photographs $\mathrm{b}-\mathrm{d}$ by Phil Lester).

we considered butterflies to be infected with $O$. elektroscirrha if their spore counts were categorised as either 2 or 3 in the above rationale. We used mixed-effects binary logistic regression to model the presence or absence of wing deformity, or parasite presence or absence, using the package lme4 (Bates et al., 2015) in $\mathrm{R}$ (R Core Team, 2020) with ggplot2 (Wickham, 2016). lme4 uses maximum likelihood to fit models. The random effect in the models was the submitting individual/family. The fixed effects were latitude, and the sex of the butterfly (male or female). Our initial models included an additional fixed effect on whether the butterfly cocoon was known to have completed development in an artificial environment (e.g. a glasshouse, porch) or it developed in a wild setting. Akaike information criterion (AIC) values were used to compare different models. For the final model, we calculated the proportion of variance explained by both fixed and random factors (the conditional $R^{2}$ value) using the $R$ package MuMIn (Bartoń, 2020).

Latitudinal gradients are correlated with variation in temperature. We explored this relationship using temperature data from the National Climate Database (CliFlo) of New Zealand's National Institute of Water and Atmospheric Research. We downloaded the average air temperature for the months of
March, April, and May 2020 (Anonymous, 2020), which encompassed the developmental period in the New Zealand autumn for our butterfly samples. Temperature stations were typically located within $10 \mathrm{~km}$ of the georeferenced sample collection.

We first used a linear regression analysis to show the relationship between latitude and average temperature, which showed a highly significant, positive relationship $(P<0.001)$ with an adjusted $R^{2}$ of 0.787 . The relationships between the likelihood of wing deformity and parasite infection and temperature mirrored that with the analyses on latitude. The fit of the relationships, as assessed by AIC values, was better for the prevalence of parasites when using latitude as an independent variable rather than temperature ( 529.25 vs. 539.88 , respectively). For wing deformities, the latitude and temperature gave similar AIC values (408.02 and 407.91, respectively). For consistency between analyses, we used latitude as the explanatory variable rather than temperature.

To confirm the identity of the parasite via molecular methods, we extracted DNA from parasite spores collected from eight monarch butterflies in sticky tape from three sampling locations (Auckland, Levin, and Christchurch). We selected samples 
with the highest parasite infections (Table S1). Under the microscope, we separated the parasite spores from the sticky tape (making sure not to include butterfly wing scales) with small tweezers and needles and placed them in a microcentrifuge tube with $1 \mathrm{ml}$ of isopropanol. The majority of spores were stuck to small pieces of tape, so they were left in isopropanol for 3 days. We then proceeded to DNA extraction using the Quick-DNA MiniPrep Kit (Zymo Research, USA). Spores were mechanically homogenised in a Precellys Evolution homogeniser (Bertin Instruments, France) in $500 \mu \mathrm{l}$ of genomic lysis buffer for two cycles of $9900 \mathrm{rpm}$ each. Samples were incubated in a heat block at $56^{\circ} \mathrm{C}$ for $2 \mathrm{~h}$. The remaining of the protocol followed the kit's manufacturer instructions. Following DNA extraction, we amplified a $558 \mathrm{bp}$ fragment of $18 \mathrm{~S}$ rRNA using the primers designed by Gao et al. (2020). Reaction volume was $50 \mu \mathrm{l}$ containing 10 ng DNA, 1x MyTaq Mix (Bioline, Australia), 5 pmol of each primer (Thermo Fisher, New Zealand), Bovine Serum Albumin (Sigma Aldrich, New Zealand), and ultra-pure, distilled water (Invitrogen, New Zealand). The PCR product was examined by agarose gel electrophoresis and purified with rSap combined with Exo 1 (New England Biolabs, USA). Sequencing was performed on an ABI 3130x1 Genetic Analyzer (Applied Biosystems, USA) at Massey Genome Service (Palmerston North, New Zealand). We aligned the forward and reverse gene sequences using the default alignment algorithm implemented in the software Geneious v. 10.2.6 (http://www.geneious.com). The resulting sequence was submitted to GenBank (GenBank accession number: MT742950).

\section{Results}

We received 564 samples of adult butterflies spanning the distribution of monarch butterflies in New Zealand (Fig. 1e). Out of these, five samples were excluded as there were no scales in the sticky tape, thus our effective sample size was 559. For samples where the butterfly sex was recorded $(n=510)$, $53.1 \%$ were female and $46.9 \%$ were male. Out of the 559 samples, $58.9 \%(n=329)$ were infected with O. elektroscirrha spores, with $38.4 \%$ of specimens being recorded as having deformed wings (134 butterflies with deformed wings out of 385 butterflies with wing status reported; Fig. S1). No parasitism was observed in our highest latitudinal location of Dunedin, while all butterfly samples were infected in the warmest and lowest latitudinal site of Doubtless Bay (Fig. 1e). Our 18S rRNA sequence confirmed that the parasite present in New Zealand is indeed $O$. elektroscirrha. Our sample matched the sequences of $O$. elektroscirrha extracted from monarch butterflies from Costa Rica and the Netherlands in the GenBank database with a similarity of $100 \%$.

Contrasting patterns of wing deformity and parasitism infection were observed. The rate of wing deformities increased with increasing latitude (Figs 1e and 2). Our logistic regression model predicted $<20 \%$ specimens would have wing deformities in the lowest latitude or warmest field sites. In the highest latitudinal sites, the prevalence of wing deformities was predicted to increase to $>80 \%$. In contrast, parasitism by $O$. elektroscirrha was shown to be lowest in the higher latitudinal localities sampled $(<10 \%)$, though increased to $>90 \%$ in the lowest latitudinal sites (Fig. 2). These counter-cline results were contrary to our expectations, in which we expected wing deformities to be correlated with parasitism rates. The average temperature in the austral autumn months (when samples were collected, from April until the end of May) declines along this latitudinal range from $17.0^{\circ} \mathrm{C}$ in Auckland, to $14.7^{\circ} \mathrm{C}$ in Wellington, to $13.5^{\circ} \mathrm{C}$ in Dunedin (Anonymous, 2020). Latitude in New Zealand is highly correlated with temperature.

Our mixed-effects logistic regression model examined how the presence or absence of parasitism was related to latitude, butterfly sex, and wing deformity. Our initial model included the categorisation of whether the butterfly cocoon was known to have completed development in an artificial environment (e.g. a glasshouse) or it developed in a wild setting. Because the rearing method was not significant in the analyses for either parasitism or wing deformities $(P \geq 0.655)$, we excluded it from the final logistic regression models. These final models incorporated the individual submitter or family as a random factor and included only the main effects of latitude and butterfly sex. For the presence or absence of spores, latitude was statistically significant with a positive slope $(z=4.933 ; \beta=0.805 \pm 0.161$; $P<0.001)$. The conditional $R^{2}$ value indicated that $75.7 \%$ of the variation in the presence or absence of spores was explained by this model. For wing deformity, latitude was also statistically significant, but with the opposing effect of a negative slope $(z=-2.965 ; \beta=-0.357 \pm 0.120 ; P=0.003)$. The conditional $R^{2}$ value indicated that $55.5 \%$ of the variation in the presence or absence of wing deformity was explained by this model. The prevalence of parasitism or wing deformity did not significantly vary with the butterfly sex $(P \geq 0.068)$.

We obtained the average air temperature for each sampling location for the autumn period. Substituting the average air temperature for latitude gave very similar results in our mixed-effects logistic regression models. For the presence or absence of spores, temperature was statistically significant with a positive slope $(z=4.418 ; \beta=1.525 \pm 0.345 ; P<0.001)$. For wing deformity, temperature was also statistically significant with the opposing effect of a negative slope $(z=-2.928$; $\beta=-0.682 \pm 0.233 ; P=0.003)$. Again, either the prevalence of parasitism or wing deformity did not vary significantly with the sex of the butterfly $(P \geq 0.079)$.

\section{Discussion}

We observed a distinct gradient in butterfly parasitism rates by $O$. elektroscirrha in New Zealand. Infection prevalence was substantially higher in the warmer environments to the north and lowest in the coolest, southernmost sites of New Zealand. High infections of this protozoan parasite can have substantial fitness costs for monarch butterflies, manifesting as a reduced mating ability, fecundity, flight ability, and adult lifespan (de Roode et al., 2019). Our results suggest that the high prevalence of parasite infections may affect butterfly fitness in warmer regions, but the low $O$. elektroscirrha prevalence in higher latitudes means that any detrimental effects from this parasite must be substantially reduced in these cooler environmental conditions. 
(a) Probability of deformed wings

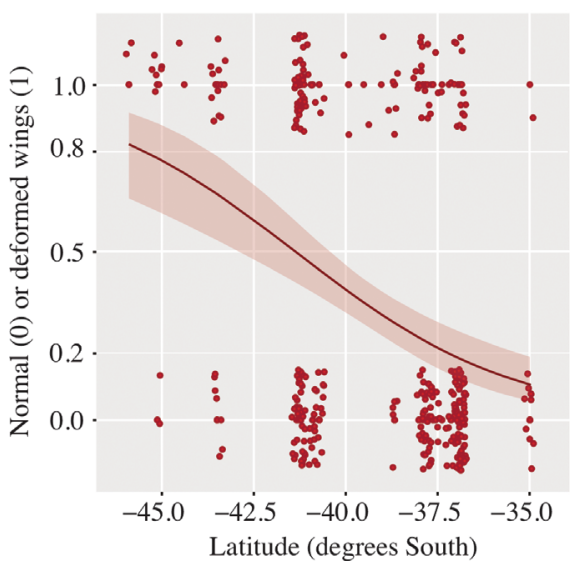

(b) Probability of $O$. elektroscirrha infection

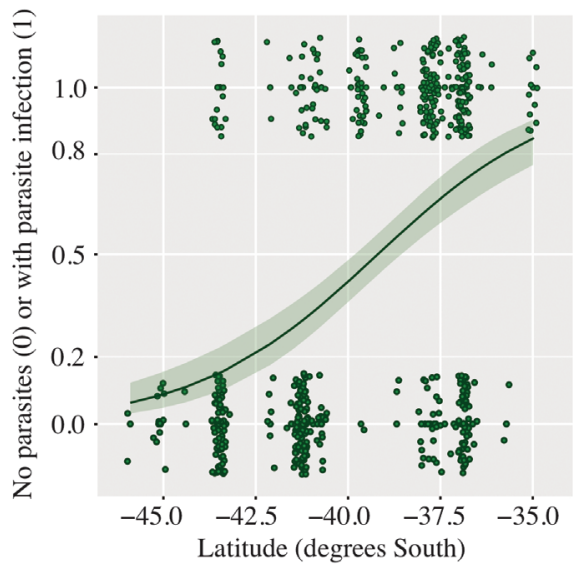

Fig. 2. Logistic regression analyses of (a) butterflies displaying normal or deformed wings, and (b) the presence or absence of O. elektroscirrha spores. All values were either 0 or 1 , but are displayed here using the 'jitter' function of ggplot 2 in order to provide an indication of sample sizes. The fitted curves are shown with $99 \%$ confidence intervals.

Butterflies at higher latitudes with cooler conditions, however, have a major fitness cost expressed as wing deformity. We hypothesise that monarch butterfly populations may perform better at intermediate latitudes and temperatures.

A latitudinal gradient in parasitism is similarly observed in populations of monarch butterflies in North America, where three distinct populations occur. A population in southeastern USA where the majority of butterflies are non-migratory and breed year-round; a population in the western coast of North America with some individuals migrating short distances to overwinter in California, where seasonal and year-round breeding sites can also be found (Satterfield et al., 2016); and a population in eastern North America that migrates from as far north as Canada to Central Mexico (Altizer et al., 2000). The population migrating from the highest latitudes has the lowest rate of parasitism by O. elektroscirrha (reviewed by de Roode et al., 2019). The year-round breeding, non-migratory populations found in the warmer areas of southern Texas, Louisiana, Mississippi, Alabama, Georgia, and Florida together with the non-migratory southern California populations have the highest parasitism rate (Satterfield et al., 2016; de Roode et al., 2019). In addition, these non-migratory populations of monarchs have also been described as susceptible to chilling injury and unable to withstand freezing (Troyer et al., 1996). The lower development threshold for monarch larvae is $11-12^{\circ} \mathrm{C}$ (Zalucki, 1982); larvae are freeze-intolerant, whereas butterfly eggs are chill-intolerant, with third instars being most sensitive to extreme cold and heat exposure (Nail et al., 2015). The increase in wing deformities with increasing latitude in our study seems likely to be related to cold temperatures. Our results from the comparatively simple tritrophic system with non-migratory butterflies in New Zealand suggest that limited cold tolerance may be a driver of parasitism rates, wing deformities, and butterfly migratory behaviour. However, given the potential for inbreeding in New Zealand monarchs due to the founder effects experienced (Pierce et al., 2014), we cannot discount the possibility that a low level of genetic diversity could be affecting the development of wings through altering mutation frequencies in the wingless gene, bacterial infections, and/or the absence of the endosymbiont Wolbachia (Pierzynowska et al., 2019). Future research studies that analyse the known causes of wing malformations in butterflies in New Zealand monarchs are indeed warranted.

In North American monarch populations, migration has been considered likely to mediate interactions between the monarch host and the O. elektroscirrha parasite (Altizer et al., 1999). The parasite-host interaction was suggested as a major force in driving seasonal migration behaviour (Altizer et al., 2000; Bradley \& Altizer, 2005; Bartel et al., 2011; Flockhart et al., 2018; de Roode et al., 2019). The use of the same butterfly habitats for long periods allows for the accumulation of the parasite infectious stages over time; migration helps the butterflies escape from the contaminated habitats. This 'migratory escape' of the parasites might play a role in the evolution of migration (Altizer $\&$ de Roode, 2015). 'Migratory culling' or the selective removal of weak, infected butterflies during their energetically costly migration could also drive the host-parasite patterns seen in North America (Bradley \& Altizer, 2005; Bartel et al., 2011). Migratory culling or migratory escape could well be evolutionary drivers of the monarch's migration behaviour in North America. A benefit of the migration would be to avoid chilling injuries resulting in the wing deformities that we observed in the New Zealand populations, which are non-migratory (Wise, 1980). The apparent intolerance of cold temperatures by the parasite that we observed may also contribute to the $O$. elektroscirrha prevalence patterns in North America and other countries. Laboratory studies rearing butterflies at different temperatures, with and without parasites, will be needed to control for the effects of migration and further understand the role of these parasites and the environment on monarch fitness.

Laboratory studies with monarch butterflies and their parasite may also enable researchers to control for other effects that could have contributed to our results. Monarch butterfly distribution is certainly influenced by the availability of host plants (Zalucki \& Rochester, 1999). Monarch butterfly density may have varied 
across our study region, which could also have influenced the abundance of the parasite. The effects of butterfly density on susceptibility to $O$. elektroscirrha have been quantified in the laboratory, with the probability of infection increasing with increasing larval density. Monarchs reared at intermediate and high densities suffered the greatest negative effects of parasitism (Lindsey et al., 2009). Variation in relative humidity, particularly dry stress as seen in Australia, might have also played a role in the outcome of the host-parasite relationship (Altizer et al., 1999).

Monarch butterfly populations have been considered to be threatened, due to a variety of factors including climate change that may result in the freezing of butterfly populations (Nail et al., 2019; Pennisi, 2021). Other researchers have examined the role of climatic factors, habitat loss, disease, and agricultural insecticide use, finding that breeding season temperature was an especially important determinant of annual variation in abundance (Thogmartin et al., 2017). Our results suggest that disease and climate might interact in a latitude-specific fashion. Conservation approaches may need to be regionally tailored to account for interacting factors, such as parasite-climate interactions. Finally, these results further highlight the benefits and immense value of the "citizen army for science" in understanding monarch butterfly biology and behaviour (Ries \& Oberhauser, 2015). Citizen science has had a long history in deciphering the migration of these butterflies (Urquhart, 1976). Our study supports the conclusion that opportunistically-collected citizen science data can transform our understanding of species distributions, phenology, and diversity gradients for these butterflies and many other species (Soroye et al., 2018).

\section{Acknowledgements}

We are extremely grateful to the passionate and enthusiastic people who submitted samples from butterflies. Thanks also to Jacqui Knight and the Moths and Butterflies of New Zealand Trust for help in advertising this research programme. We thank James Baty, Jana Dobelmann, John Haywood, Matt Howse and Rose McGruddy for comments. Thanks to Lara Shepherd for providing information and resources about milkweed distribution in New Zealand. We also thank the Associate Editor and two anonymous reviewers for their useful comments that substantially improved our original manuscript.

The authors have no conflict of interest to declare.

\section{Author contributions}

P.J.L. conceived and coordinated the study. Both authors analysed the samples and wrote the manuscript. M.B. conducted the genetics lab work and categorised the spore counts.

\section{Data availability statement}

The data that supports the findings of this study are available in the supplementary material of this article.

\section{Supporting Information}

Additional supporting information may be found online in the Supporting Information section at the end of the article.

Table S1: The complete dataset used in our analysis. Data on sample location, parasitism, butterfly sex and wing deformities.

Fig. S1: Graph showing the spore samples that fell into each of the four categories for spore counts.

\section{References}

Albrectsen, B.R., Witzell, J., Robinson, K.M., Wulff, S., Luquez, V.M.C., Ågren, R. et al. (2010) Large scale geographic clines of parasite damage to Populus tremula L. Ecography, 33, 483-493.

Altizer, S. \& de Roode, J.C. (2015) Monarchs and their debilitating parasites: Immunity, migration and medicinal plant use. Monarchs in a Changing World: Biology and Conservation of an Iconic Insect (ed. by K. Oberhauser, S. Altizer and K. Nail). Cornell University Press, Ithaca, NY.

Altizer, S.M., Oberhauser, K.S. \& Brower, L.P. (1999) Host migration and the prevalence of the protozoan parasite, Ophryocystis elektroscirrha. Natural populations of Adult Monarch Butterflies. The 1997 North American Conference on the Monarch Butterfly (ed. by J. Hoth, L. Merino, K. Oberhauser, I. Pisanty, S. Price and T. Wilkinson), pp. 165-176. Commission for Environmental Cooperation, Montreal, Canada.

Altizer, S.M., Oberhauser, K.S. \& Brower, L.P. (2000) Associations between host migration and the prevalence of a protozoan parasite in natural populations of adult monarch butterflies. Ecological Entomology, 25, 125-139.

Anonymous (2020) CliFlo: NIWA's National Climate Database on the Web, Vol. 2020. National Institute of Water and Atmospheric Research, Ltd, Auckland, New Zealand.

Bartel, R.A., Oberhauser, K.S., de Roode, J.C. \& Altizer, S.M. (2011) Monarch butterfly migration and parasite transmission in eastern North America. Ecology, 92, 342-351.

Bartoń, K. (2020) MuMIn: multi-model inference. $R$ package version, 1, 17. https://CRAN.R-project.org/package=MuMIn.

Bates, D., Maechler, M., Bolker, B. \& Walker, S. (2015) Fitting linear mixed-effects models using lme4. Journal of Statistical Software, 67, $1-48$.

Bradley, C.A. \& Altizer, S. (2005) Parasites hinder monarch butterfly flight: implications for disease spread in migratory hosts. Ecology Letters, 8, 290-300.

Calvete, C., Estrada, R., Lucientes, J., Estrada, A. \& Telletxea, I. (2003) Correlates of helminth community in the red-legged partridge (Alectoris rufa L.) in Spain. Journal of Parasitology, 89, 445-451.

Clarke, A.R. \& Zalucki, M.P. (2004) Monarchs in Australia: on the winds of a storm? Biological Invasions, 6, 123-127.

Corby-Harris, V. \& Promislow, D.E.L. (2008) Host ecology shapes geographical variation for resistance to bacterial infection in Drosophila melanogaster. Journal of Animal Ecology, 77, 768-776.

Cumming, G.S. (2000) Using habitat models to map diversity: pan-African species richness of ticks (Acari: Ixodida). Journal of Biogeography, 27, 425-440.

Davis, A.K. \& Rendon-Salinas, E. (2010) Are female monarch butterflies declining in eastern North America? Evidence of a 30-year change in sex ratios at Mexican overwintering sites. Biology Letters, 6, 45-47.

Davis, A.K., Altizer, S. \& Friedle, E. (2004) A non-destructive, automated method of counting spores of Ophryocystis elektroscirrha 
(Neogregarinorida: Ophryocystidae) in infected monarch butterflies (Lepidoptera: Nymphalidae). Florida Entomologist, 87, 231-234.

Dawson, R.D., Hillen, K.K. \& Whitworth, T.L. (2005) Effects of experimental variation in temperature on larval densities of parasitic Protocalliphora (Diptera: Calliphoridae) in nests of tree swallows (Passeriformes: Hirundinidae). Environmental Entomology, 34, $563-568$.

Dunn, R.R., Davies, T.J., Harris, N.C. \& Gavin, M.C. (2010) Global drivers of human pathogen richness and prevalence. Proceedings of the Royal Society B: Biological Sciences, 277, 2587-2595.

Flockhart, D.T.T., Dabydeen, A., Satterfield, D.A., Hobson, K.A., Wassenaar, L.I. \& Norris, D.R. (2018) Patterns of parasitism in monarch butterflies during the breeding season in eastern North America. Ecological Entomology, 43, 28-36.

Gao, K., Muijderman, D., Nichols, S., Heckel, D.G., Wang, P., Zalucki, M.P. et al. (2020) Parasite-host specificity: a cross-infection study of the parasite Ophryocystis elektroscirrha. Journal of Invertebrate Pathology, 170, 107328.

Gibbs, G.W. (1980) New Zealand Butterflies. Identification and Natural History. William Collins Publishers, Ltd., Auckland, New Zealand.

Gibbs, G. (2013) The Monarch Butterfly in New Zealand. The Caxton Press, Christchurch, New Zealand.

Harvell, C.D., Mitchell, C.E., Ward, J.R., Altizer, S., Dobson, A.P., Ostfeld, R.S. et al. (2002) Climate warming and disease risks for terrestrial and marine biota. Science, 296, 2158-2162.

Leong, K.L.H., Yoshimura, M.A. \& Kaya, H.K. (1997) Occurrence of a neogregarine protozoan, Ophryocystis elektroscirrha McLaughlin and Myers, in populations of monarch and queen butterflies. The Pan-Pacific Entomologist, 73, 49-51.

Li, N., Chen, Q., Zhu, J., Wang, X., Huang, J.-B. \& Huang, G.-H. (2017) Seasonal dynamics and spatial distribution pattern of Parapoynx crisonalis (Lepidoptera: Crambidae) on water chestnuts. PLoS One, 12, e0184149.

Lindsey, E., Mehta, M., Dhulipala, V., Oberhauser, K. \& Altizer, S. (2009) Crowding and disease: effects of host density on response to infection in a butterfly-parasite interaction. Ecological Entomology, 34, 551-561.

Loiseau, C., Iezhova, T., Valkiunas, G., Chasar, A., Hutchinson, A., Buermann, W. et al. (2010) Spatial variation in haemosporidian parasite infection in African rainforest bird species. Journal of Parasitology, 96, 21-29.

Majewska, A.A., Schneider, A., Simms, S., Altizer, S. \& Hall, R.J. (2019) Multiple transmission routes sustain high prevalence of a virulent parasite in a butterfly host. Proceedings of the Royal Society B: Biological Sciences, 286, 20191630.

McLaughlin, R.E. \& Myers, J. (1970) Ophryocystis elektroscirrha sp. n., a neogregarine pathogen of the monarch butterfly Danaus plexippus (L.) and the Florida queen butterfly D. gilippus berenice Cramer. Journal of Protozoology, 17, 300-305.

Musgrave, K., Bartlow, A.W. \& Fair, J.M. (2019) Long-term variation in environmental conditions influences host-parasite fitness. Ecology and Evolution, 9, 7688-7703.

Nail, K.R., Batalden, R.V. \& Oberhauser, K.S. (2015) What's too hot and what's too cold. Monarchs in a Changing World: Biology and Conservation of an Iconic Insect (ed. by K. S. Oberhauser, K. R. Nail and S. M. Altizer), pp. 99-108. Cornell University Press, Ithaca, NY.

Nail, K.R., Drizd, L. \& Voorhies, K.J. (2019) Butterflies across the globe: a synthesis of the current status and characteristics of monarch (Danaus plexippus) populations worldwide. Frontiers in Ecology and Evolution, 7, 362.

Nunn, C.L., Altizer, S.M., Sechrest, W. \& Cunningham, A.A. (2005) Latitudinal gradients of parasite species richness in primates. Diversity and Distributions, 11, 249-256.
Pennisi, E. (2021) Are monarchs in trouble? Science, 371, 219-220.

Pierce, A.A., Zalucki, M.P., Bangura, M., Udawatta, M., Kronforst, M.R., Altizer, S. et al. (2014) Serial founder effects and genetic differentiation during worldwide range expansion of monarch butterflies. Proceedings of the Royal Society B: Biological Sciences, 281, 2230. https://doi.org/10.1098/rspb.2014.2230.

Pierzynowska, K., Skowron Volponi, M. \& Wegrzyn, G. (2019) Multiple factors correlating with wing malformations in the population of Parnassius apollo (Lepidoptera: Papilionidae) restituted from a low number of individuals: a mini review. Insect Science, 26, 380-387.

Poulin, R. (2007) Evolutionary Ecology of Parasites. Princeton University Press, Princeton, NJ.

$\mathrm{R}$ Core Team (2020) R: A language and environment for statistical computing, Version 4.0.2. Vienna, Austria: R Foundation for Statistical Computing.

Ries, L. \& Oberhauser, K. (2015) A citizen army for science: quantifying the contributions of citizen scientists to our understanding of monarch butterfly biology. Bioscience, 65, 419-430.

Rohde, K. (1993) Ecology of Marine Parasites: An Introduction to Marine Parasitology, 2nd edn. CABI International, Wallingford, U.K.

Rohde, K. (1999) Latitudinal gradients in species diversity and Rapoport's rule revisited: a review of recent work and what can parasites teach us about the causes of the gradients? Ecography, 22, $593-613$

Rohde, K. (2002) Ecology and biogeography of marine parasites. Advances in Marine Biology, 43, 1-86.

Rohde, K. \& Heap, M. (1998) Latitudinal differences in species and community richness and in community structure of metazoan endoand ectoparasites of marine teleost fish. International Journal for Parasitology, 28, 461-474.

de Roode, J.C., Gold, L.R. \& Altizer, S. (2007) Virulence determinants in a natural butterfly-parasite system. Parasitology, 134, 657-668.

de Roode, J.C., Yates, A.J. \& Altizer, S. (2008a) Virulence-transmission trade-offs and population divergence in virulence in a naturally occurring butterfly parasite. Proceedings of the National Academy of Sciences of the U.S.A., 105, 7489-7494.

de Roode, J.C., Pedersen, A.B., Hunter, M.D. \& Altizer, S. (2008b) Host plant species affects virulence in monarch butterfly parasites. Journal of Animal Ecology, 77, 120-126.

de Roode, J.C., Chi, J., Rarick, R.M. \& Altizer, S. (2009) Strength in numbers: high parasite burdens increase transmission of a protozoan parasite of monarch butterflies (Danaus plexippus). Oecologia, 161, $67-75$.

de Roode, J.C., Altizer, S. \& Hunter, M.D. (2019) Multi-trophic interactions and migration behaviour determine the ecology and evolution of parasite infection in monarch butterflies. Wildlife Disease Ecology: Linking Theory to Data and Application (ed. by K. Wilson, A. Fenton and D. M. Tompkins), pp. 480-510. Cambridge University Press, Cambridge, U.K.

Satterfield, D., Maerz, J. \& Altizer, S. (2015) Loss of migratory behavior supports high parasite prevalence in a butterfly host. Proceedings of the Royal Society Series B: Biological Sciences, 1801, 20141734.

Satterfield, D.A., Villablanca, F.X., Maerz, J.C. \& Altizer, S. (2016) Migratory monarchs wintering in California experience low infection risk compared to monarchs breeding year-round on non-native milkweed. Integrative and Comparative Biology, 56, 343-352.

Scharsack, J.P., Franke, F., Erin, N.I., Kuske, A., Büscher, J., Stolz, H. et al. (2016) Effects of environmental variation on host-parasite interaction in three-spined sticklebacks (Gasterosteus aculeatus). Zoology, 119, 375-383. 
Soroye, P., Ahmed, N. \& Kerr, J.T. (2018) Opportunistic citizen science data transform understanding of species distributions, phenology, and diversity gradients for global change research. Global Change Biology, 24, 5281-5291.

Thogmartin, W.E., Wiederholt, R., Oberhauser, K., Drum, R.G., Diffendorfer, J.E., Altizer, S. et al. (2017) Monarch butterfly population decline in North America: identifying the threatening processes. Royal Society Open Science, 4, 170760.

Timi, J.T. (2003) Parasites of argentine anchovy in the south-west Atlantic: latitudinal patterns and their use for discrimination of host populations. Journal of Fish Biology, 63, 90-107.

Troyer, H.L., Burks, C.S. \& Lee, R.E. (1996) Phenology of cold hardiness in reproductive and migrant monarch butterflies (Danaus plexippus) in Southwest Ohio. Journal of Insect Physiology, 42, 633-642.

Urquhart, F.A. (1976) Found at last: the monarch's winter home. National Geographic, 150, 160-173.

Webberley, K.M., Tinsley, M.C., Sloggett, J.J., Majerus, M.E.N. \& Hurst, G.D.D. (2006) Spatial variation in the incidence of a sexually transmitted parasite of the ladybird beetle Adalia bipunctata (Coleoptera: Coccinellidae). European Journal of Entomology, 103, 793-797.

Wickham, H. (2016) ggplot2: Elegant Graphics for Data Analysis. Springer-Verlag, New York.

Wise, K.A.J. (1980) Monarch butterfly dispersal in New Zealand. Records of the Auckland Institute and Museum, 17, 157-173.
Wolinska, J., Seda, J., Koerner, H., Smilauer, P. \& Petrusek, A. (2011) Spatial variation of Daphnia parasite load within individual water bodies. Journal of Plankton Research, 33, 1284-1294.

Young, V. (2020) Citizen scientists enlisted to help monarch butterflies. Radio New Zealand, Wellington, New Zealand. https://www.rnz .co.nz/national/programmes/ninetonoon/audio/2018746121/citizenscientists-enlisted-to-help-monarch-butterflies.

Zalucki, M.P. (1982) Temperature and rate of development in Danaus plexippus L. and D. chrysippus L. (Lepidoptera: Nymphalidae). Australian Journal of Entomology, 21, 241-246.

Zalucki, M.P. \& Clarke, A.R. (2004) Monarchs across the Pacific: the Columbus hypothesis revisited. Biological Journal of the Linnean Society, 82, 111-121.

Zalucki, M.P. \& Rochester, W.A. (1999) Estimating the effect of climate on the distribution and abundance of Danaus plexippus: A tale of two continents. The 1997 North American Conference on the Monarch Butterfly (ed. by J. Hoth, L. Merino, K. Oberhauser, I. Pisanty, S. Price and T. Wilkinson), pp. 151-163. Commission for Environmental Cooperation, Montreal, Canada.

Zilberstein, D. \& Shapira, M. (1994) The role of $\mathrm{pH}$ and temperature in the development of Leishmania parasites. Annual Review of Microbiology, 48, 449-470.

Accepted 28 May 2021

First published online 12 June 2021

Associate Editor: Toomas Tammaru 